\title{
Radiotherapy treatment planning with contrast-enhanced computed tomography: feasibility of dual-energy virtual unenhanced imaging for improved dose calculations
}

Sachiko Yamada ${ }^{1,2^{*}}$, Takashi Ueguchi ${ }^{3,4}$, Toshiyuki Ogata ${ }^{5}$, Hirokazu Mizuno ${ }^{1}$, Ryota Ogihara', Masahiko Koizumi², Takeshi Shimazu ${ }^{6}$, Kenya Murase ${ }^{2}$ and Kazuhiko Ogawa ${ }^{7}$

\begin{abstract}
Background: In radiotherapy treatment planning, intravenous administration of an iodine-based contrast agent during computed tomography $(\mathrm{CT})$ improves the accuracy of delineating target volumes. However, increased tissue attenuation resulting from the high atomic number of iodine may result in erroneous dose calculations because the contrast agent is absent during the actual procedure. The purpose of this proof-of-concept study was to present a novel framework to improve the accuracy of dose calculations using dual-energy virtual unenhanced $\mathrm{CT}$ in the presence of an iodine-based contrast agent.

Methods: Simple phantom experiments were designed to assess the feasibility of the proposed concept. By utilizing a "second-generation" dual-source CT scanner equipped with a tin filter for improved spectral separation, four CT datasets were obtained using both a water phantom and an iodine phantom: "true unenhanced" images with attenuation values of $2 \pm 11$ Hounsfield Units (HU), "enhanced" images with attenuation values of $274 \pm 23 \mathrm{HU}$, and two series of "virtual unenhanced" images synthesized from dual-energy scans of the iodine phantom, each with a different combination of tube voltages. Two series of virtual unenhanced images demonstrated attenuation values of $12 \pm 29 \mathrm{HU}$ (with $80 \mathrm{kVp} / 140 \mathrm{kVp}$ ) and $34 \pm 10 \mathrm{HU}$ (with $100 \mathrm{kVp} / 140 \mathrm{kVp}$ ) after removing the iodine component from the contrast-enhanced images. Dose distributions of the single photon beams calculated from the enhanced images and two series of virtual unenhanced images were compared to those from true unenhanced images as a reference.

Results: The dose distributions obtained from both series of virtual unenhanced images were almost equivalent to that from the true unenhanced images, whereas the dose distribution obtained from the enhanced images indicated increased beam attenuation caused by the high attenuation characteristics of iodine. Compared to the reference dose distribution from the true unenhanced images, the dose distribution pass rates from both series of virtual unenhanced images were greater than $90 \%$, while those from the enhanced images were less than approximately 50-60\%.
\end{abstract}

Conclusions: Dual-energy virtual unenhanced CT improves the accuracy of dose distributions in radiotherapy treatment planning by removing the iodine component from contrast-enhanced images.

Keywords: Radiotherapy treatment planning, Dual-energy $C T$, Virtual unenhanced $C T$, Dose calculation, lodine contrast agent, Tissue attenuation, $C T$ number, Dual-source $C T$, Tin filter

\footnotetext{
* Correspondence: yamada@hp-rad.med.osaka-u.ac.jp

'Department of Radiology, Osaka University Hospital, 2-15 Yamadaoka, Suita,

Osaka 565-0871, Japan

${ }^{2}$ Department of Medical Physics and Engineering, Osaka University Graduate

School of Medicine, 1-7 Yamadaoka, Suita, Osaka 565-0871, Japan

Full list of author information is available at the end of the article
} 


\section{Background}

The use of computed tomography (CT) is now an established procedure in modern radiotherapy treatment planning. Cross-sectional CT data allow for the accurate delineation of surface contours and internal structures including target and critical organs. In addition, the pixel values, i.e., the CT numbers in Hounsfield Units (HU), provide tissue electron density information, which allows a pixel-by-pixel correction for tissue inhomogeneities when calculating dose distributions. Although other imaging modalities are becoming important tools in treatment planning, CT is still the fundamental and primary modality.

An inherent disadvantage of $\mathrm{CT}$ is its relatively lower contrast between lesions and surrounding tissue or vessels. Thus, diagnostic $\mathrm{CT}$ often requires the intravenous administration of an iodine-based contrast agent to enhance lesion-to-tissue contrast. Because the accurate delineation of target volumes (and organs at risk) is crucial in radiotherapy treatment planning, the use of an iodine-based contrast agent is necessary in situations in which contrast is likely to improve the accuracy of the target or normal structure delineations. However, there is a concern that the introduction of contrast agent may result in erroneous dose calculations [1]. In an actual treatment situation, the patient does not receive contrast agent, and hence the tissue density during treatment will differ from that during a contrast-enhanced CT scan. Although several clinical studies on head and neck [2], lung [3], and prostate cancers [4] have shown that contrast media had little influence on dose calculations, this issue is still debated [5-7].

A recent advance in the field of $\mathrm{CT}$ was the introduction of dual-energy CT. Dual-energy CT acquires two different datasets with different tube voltages (i.e., beam energies). Within the range of photon energies in $\mathrm{CT}$, the CT numbers of soft tissues are almost independent of beam energy. In contrast, $\mathrm{CT}$ numbers of high atomic number materials such as iodine are noticeably energydependent. Thus, materials with a high atomic number can be further differentiated from soft tissues by applying different beam energies and analyzing the differences in attenuation [8]. This principle allows clinicians to reconstruct "virtual unenhanced" images from iodine contrast-enhanced scans [9-15]. The attenuation differences of iodine between two energies can be utilized to produce an "iodine map", which can be subsequently removed from images to create virtual unenhanced images. Thus, virtual unenhanced images theoretically provide $\mathrm{CT}$ numbers equivalent to those of true unenhanced images. It should be noted that the key to reliable virtual unenhanced imaging lies on both the spectral separation between low- and high-energy beams, and low image noise. These two fundamental requirements can be accomplished by the "second generation" dual-source CT scanner. The scanner utilizes two distinct X-ray sources with corresponding detectors at an angle separation of $95^{\circ}$. The two sources can be simultaneously operated at different tube voltages. Importantly, this feature has the capability of applying an additional tin filter for a highenergy beam. The filter is highly advantageous because it narrows the spectrum, which results in higher dose efficiency, less beam hardening, and improved spectral separation between the low- and high-energy beams $[16,17]$. The improved spectral separation allows for tube operation at a combined $100 \mathrm{kVp}$ and $140 \mathrm{kVp}$ rather than the conventional dual-energy combination of $80 \mathrm{kVp}$ and 140 $\mathrm{kVp}$ [17], and thus can reduce image noise. Furthermore, iodine removal is more accurate with the filter than without it when the subject adjoins a high attenuation material (e.g. bone, contrast-enhanced large vessels, etc.) [13].

Using this promising technique under iodine contrast enhancement, we propose a novel strategy for improved radiation treatment planning. In this scheme, dose calculations can be performed using synthesized virtual unenhanced CT images, while the delineation of the target and critical organs can be conducted using the original contrast-enhanced CT images (Figure 1). The purpose of this preliminary study was to investigate the feasibility of our proposed scheme in simple phantom experiments using the second-generation dual-source CT scanner.

\section{Methods}

This study comprised two phantom experiments. The first experiment assessed dual-energy virtual unenhanced attenuation values of iodine solutions under two different combinations of tube voltages. The second experiment investigated the feasibility of dose calculation using virtual unenhanced imaging. All experiments were conducted using a dual-source CT scanner (SOMATOM Definition Flash, Siemens AG, Erlangen, Germany). A dedicated application "Liver VNC" (Siemens) was utilized to reconstruct virtual unenhanced images.

\section{Assessment of virtual unenhanced attenuation values}

We prepared a phantom comprising eight polypropylene syringes, each containing a $10-\mathrm{mL}$ mixture of water and an iodine-based contrast agent (Iopamidol, $300 \mathrm{mgI} / \mathrm{mL}$ ) at different dilutions $(4.50 \%, 2.00 \%, 1.00 \%, 0.50 \%, 0.25 \%$, $0.13 \%, 0.06 \%$, and $0.00 \%$ by weight). The phantom was placed at the isocenter of the CT scanner, and scanned using both single-energy and dual-energy helical modes. For the single-energy scan, only one X-ray tube was operated at $120 \mathrm{kVp}$ and $100 \mathrm{mAs}$. For the dual-energy scan, two combinations of tube settings, each using the tin filter for the high-energy beam, were tested: (i) 80 $\mathrm{kVp}$ with $100 \mathrm{mAs}$ and $140 \mathrm{kVp}$ with $39 \mathrm{mAs}$ (referred to as the "80/Sn140-kVp protocol"); and (ii) $100 \mathrm{kVp}$ 


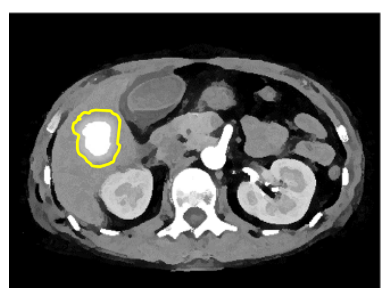

Contrast-enhanced CT for contouring target volumes.

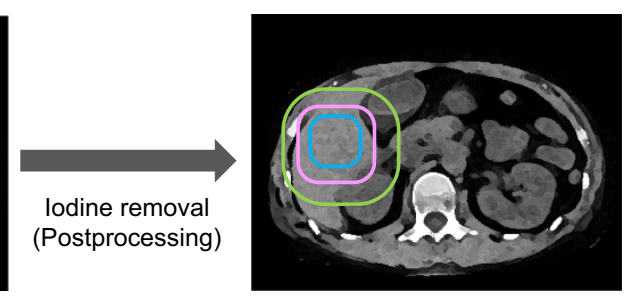

Virtual unenhanced CT for dose calculation.

Figure 1 Schematic of the proposed concept. Dose calculations can be performed using synthesized virtual unenhanced CT images, while the delineation of the target and critical organs can be conducted using the original contrast-enhanced CT images.

with $100 \mathrm{mAs}$ and $140 \mathrm{kVp}$ with $77 \mathrm{mAs}$ (referred to as the "100/Sn140-kVp protocol"). The slice thickness and reconstructed field-of-view for both scans were $5 \mathrm{~mm}$ and $150 \mathrm{~mm}$, respectively. The images were reconstructed with standard body kernels (B35f for single-energy scans and D26f for dual-energy scans). Two series of virtual unenhanced images (i.e., 80/Sn140-kVp and 100/Sn140$\mathrm{kVp}$ protocols) were synthesized from the corresponding dual-energy data. In addition, "mixed" images were synthesized using a linear weighting of the low- and high- $k V p$ images. In diagnostic dual-energy applications, the mixed images are used as representative of dual-energy data, because they are similar to traditional single-energy (i.e., 120 $\mathrm{kVp}$ ) images. Circular regions-of-interest (ROIs) were positioned within the syringes to obtain mean attenuation values in $\mathrm{HU}$. The size of the ROIs was chosen to encompass an approximately $80 \%$ area of the syringes. The scans were repeated three times, and the mean attenuation values and standard deviation of the three measurements were calculated for comparisons.

Feasibility of dose calculation using virtual unenhanced CT Because this was a pilot study, a simple phantom experiment was designed to assess the feasibility of dose calculations on virtual unenhanced CT images. Two polypropylene containers (250 mm width, $175 \mathrm{~mm}$ height, and $350 \mathrm{~mm}$ depth) filled either with distilled water (referred to as the "water phantom") or diluted (3\%) Iopamidol contrast agent (referred to as the "iodine phantom") were prepared and imaged to generate four CT datasets. First, the water phantom was scanned in single-energy mode, providing the "true unenhanced" images. Second, the iodine phantom was scanned in single-energy mode, providing the "enhanced" images. Third, the iodine phantom was scanned in dual-energy mode with the $80 / \mathrm{Sn} 140-\mathrm{kVp}$ protocol, providing the "virtual unenhanced (80/Sn140$\mathrm{kVp}$ )" images. Finally, the iodine phantom was scanned in dual-energy mode with the $100 / \mathrm{Sn} 140-\mathrm{kVp}$ protocol, providing the "virtual unenhanced (100/Sn140-kVp)" images. Each phantom was positioned on the patient table and scanned at the isocenter of the CT scanner. The imaging parameters were the same as those described above, except that the slice thickness was $2.5 \mathrm{~mm}$ and the displayed field-of-view was $330 \mathrm{~mm}$.

Attenuation values of the phantoms depicted in the four datasets were measured using ROI analysis at the central slice. The mean and standard deviation of the attenuation values for each image were obtained from a rectangular ROI positioned to encompass greater than an $80 \%$ area of the phantom, but inside the outer edges. Furthermore, attenuation profiles passing from the top to the bottom of the phantom were obtained (adjacent ten profile curves were measured and then averaged to reduce fluctuations due to noise) to evaluate attenuation values outside the ROI (especially at the bottom of the phantom where iodine sedimentation is likely to occur), although we took care to uniformly dilute the contrast agent during preparation of the iodine phantom. Figure 2 shows a photograph of the phantom and its corresponding CT images, detailing both the ROI setting for attenuation measurements and the reference line for profile curve measurements.

These four CT datasets were transferred to a Pinnacle ${ }^{3}$ treatment planning system (version $8.0 \mathrm{~m}$; Philips, Madison, WI, USA) for dose calculations. The dose distribution of a single photon beam from a Siemens Oncor Impression Plus system was calculated using a collapsed cone convolution algorithm with a dose grid size of $2 \mathrm{~mm}$. Two beam energies (4 MV and $10 \mathrm{MV})$ and two field sizes $\left(4 \times 4 \mathrm{~cm}^{2}\right.$ and $10 \times 10 \mathrm{~cm}^{2}$ ) were tested for each dataset. The beam was planned to deliver 100 monitor units at a $10-\mathrm{cm}$ depth at the center of the phantom. Although the phantom was carefully prepared, it was impossible to avoid introducing small air bubbles at the top of the phantom. Therefore, the posterior field was chosen to minimize the effects of these small bubbles on the dose calculations. The dose distributions obtained from the enhanced and virtual unenhanced (both the $80 / \mathrm{Sn} 140-\mathrm{kVp}$ and 100/ Sn140-kVp protocols) images were quantitatively compared to those from the true unenhanced images using MapCHECK (Sun Nuclear, Melbourne, FL, USA) analysis software. For the analysis, the calculated planar dose 

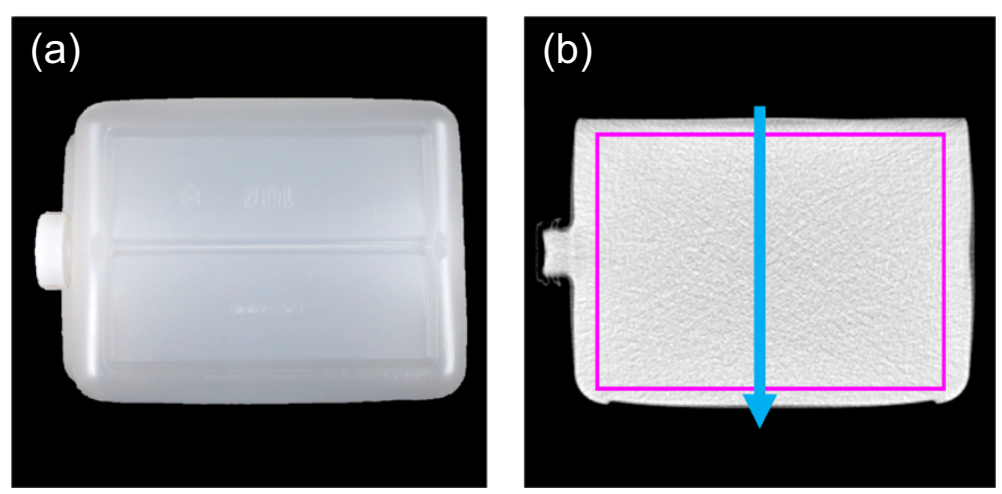

Figure 2 The phantom used in the second experiment. Photograph (a) and CT image (b) of the polypropylene container. The purple rectangle and blue arrow in (b) illustrate the region of interest for attenuation measurements and position of attenuation profile measurements, respectively.

distribution in the sagittal on-axis plane for each dataset was imported into the software; the distribution obtained from the true unenhanced images was used as a reference, and the remaining three were the subjects being tested. The assessment was performed based on the percentdose-difference and distance-to-agreement at a 10\% dose threshold. A set of acceptance criteria ( $2 \%$ and $2 \mathrm{~mm}$ ) satisfying the guideline [18] was used, and the percentage of points passing the acceptance criteria was evaluated.

\section{Results}

Figure 3 shows the results of attenuation measurements for the iodine syringes on the single-energy, and the dual-energy (100/Sn140-kVp protocol) mixed and virtual unenhanced images. The exact attenuation values including both the $80 / \mathrm{Sn} 140-\mathrm{kVp}$ and $100 / \mathrm{Sn} 140-\mathrm{kVp}$ protocols are listed in Table 1. A range of iodine concentrations

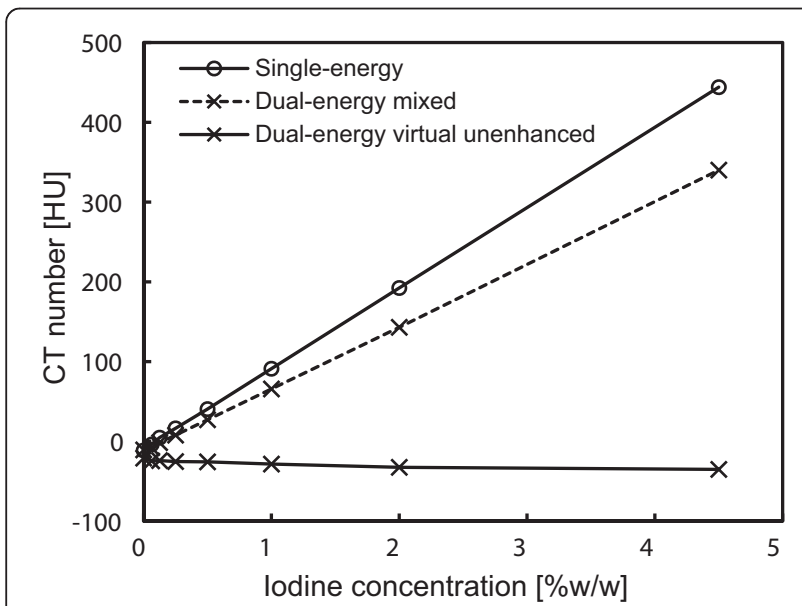

Figure 3 Attenuation measurements of iodine solutions at different iodine concentrations. Single-energy images were acquired at a tube voltage of $120 \mathrm{kVp}$. Mixed images and virtual unenhanced images were synthesized from dual-energy $(100 \mathrm{kVp}$ and $140 \mathrm{kVp}$ with a tin filter) data. tested in this experiment provided attenuation values up to approximately $440 \mathrm{HU}$ under the single-energy scan. The virtual unenhanced attenuation values using this range of iodine concentrations were reasonably stable and were close to both the single-energy and dual-energy mixed attenuation values at a $0 \%$ iodine concentration.

Figure 4 shows representative (a) true unenhanced, (b) enhanced, (c) virtual unenhanced (80/Sn140-kVp), and (d) virtual unenhanced $(100 / \mathrm{Sn} 140-\mathrm{kVp})$ images of the phantom. All the CT images were displayed at fixed window settings (window level, $50 \mathrm{HU}$; window width, 600 $\mathrm{HU})$, allowing us to compare the image contrast between the four images, visualize the effect of tube voltage settings on virtual unenhanced images ( $c$ and $d$ ), and visually confirm the presence or absence of iodine sedimentation (b). The virtual unenhanced image with the $80 / \mathrm{Sn} 140-\mathrm{kVp}$ protocol showed higher image noise and dark-band artifact, while the image obtained with the $100 / \mathrm{Sn} 140-\mathrm{kVp}$ protocol showed more uniform attenuation and less image noise. No obvious iodine sedimentation was found in the enhanced image (b). Figure 5 shows the attenuation profiles of the phantom. The profile from the enhanced image (red line) indicates that the iodine was almost uniformly distributed inside the phantom because the profile is almost flat, although the profile was slightly cupped due to beam hardening. The attenuation values of the phantoms depicted in the true unenhanced, enhanced, and virtual unenhanced images are listed in Table 2. The mean attenuation values of the iodine phantom on the virtual unenhanced images were lower $(11.6 \pm 29.2 \mathrm{HU}$ with the $80 / \mathrm{Sn} 140-\mathrm{kVp}$ protocol and 34.1 $\pm 9.9 \mathrm{HU}$ with the 100/ Sn140-kVp protocol) than those on the enhanced image $(274.3 \pm 22.5 \mathrm{HU})$.

Figures 6, 7 and 8 compare the four representative planar dose distributions $(\mathrm{a}-\mathrm{d})$, planned at beam energies of either $10 \mathrm{MV}$ (Figure 6) or $4 \mathrm{MV}$ (Figures 7 and 8), and field sizes of either $10 \times 10 \mathrm{~cm}^{2}$ (Figures 6 and 7) or $4 \times 4 \mathrm{~cm}^{2}$ (Figure 8), obtained from the true unenhanced, enhanced, 
Table 1 Attenuation measurements of iodine solutions at different iodine concentrations

\begin{tabular}{|c|c|c|c|c|c|}
\hline \multirow{3}{*}{$\begin{array}{c}\text { lodine concentration } \\
{[\% \mathrm{w} / \mathrm{w}]}\end{array}$} & \multicolumn{5}{|c|}{ Attenuation value $[\mathrm{HU}]$} \\
\hline & \multirow{2}{*}{$\begin{array}{l}\text { Single-energy } \\
(120 \mathrm{kVp})\end{array}$} & \multicolumn{2}{|c|}{ Dual-energy (80 kVp/Sn140 kVp) } & \multicolumn{2}{|c|}{ Dual-energy $(100 \mathrm{kVp} / \mathrm{Sn} 140 \mathrm{kVp})$} \\
\hline & & Mixed & Virtual unenhanced & Mixed & Virtual unenhanced \\
\hline 0.00 & $-11.3 \pm 1.3$ & $-10.4 \pm 0.1$ & $-23.0 \pm 1.0$ & $-10.7 \pm 0.6$ & $-21.0 \pm 0.6$ \\
\hline 0.06 & $-3.9 \pm 0.5$ & $-6.9 \pm 0.1$ & $-26.8 \pm 0.9$ & $-8.0 \pm 0.4$ & $-24.5 \pm 1.5$ \\
\hline 0.13 & $4.7 \pm 0.5$ & $0.1 \pm 0.2$ & $-25.1 \pm 0.8$ & $-1.6 \pm 0.4$ & $-24.1 \pm 0.7$ \\
\hline 0.25 & $16.4 \pm 0.4$ & $11.1 \pm 1.1$ & $-26.6 \pm 1.8$ & $7.9 \pm 0.8$ & $-25.3 \pm 1.1$ \\
\hline 0.50 & $40.5 \pm 0.5$ & $33.5 \pm 0.2$ & $-25.8 \pm 1.1$ & $27.2 \pm 0.4$ & $-25.7 \pm 1.1$ \\
\hline 1.00 & $91.1 \pm 0.4$ & $78.5 \pm 0.4$ & $-27.1 \pm 1.1$ & $65.8 \pm 0.6$ & $-28.4 \pm 0.4$ \\
\hline 2.00 & $192.3 \pm 0.3$ & $168.7 \pm 0.3$ & $-29.1 \pm 0.3$ & $143.0 \pm 0.3$ & $-32.5 \pm 1.3$ \\
\hline 4.50 & $444.1 \pm 0.7$ & $397.9 \pm 0.4$ & $-28.7 \pm 0.3$ & $340.1 \pm 0.3$ & $-35.1 \pm 1.9$ \\
\hline
\end{tabular}

Note: Attenuation values are mean \pm standard deviation from three measurements.

and virtual unenhanced (both the $80 / \mathrm{Sn} 140-\mathrm{kVp}$ and $100 /$ Sn140-kVp protocols) CT images. The dose difference (i.e. error) maps derived between the tested (enhanced or virtual unenhanced) and reference (true unenhanced) dose distributions are also shown $(\mathrm{e}-\mathrm{g})$. In each condition (Figures 6, 7 and 8), the dose distributions obtained from both the virtual unenhanced images were quite similar to that generated from the true unenhanced images, whereas the dose distribution obtained from the enhanced image indicated increased beam attenuation. Using the enhanced images, errors in dose calculations increased as the beam passed from the bottom to the top of the iodine phantom. In contrast, the errors were almost entirely 0 cGy when the virtual unenhanced images, especially the $100 /$ Sn140-
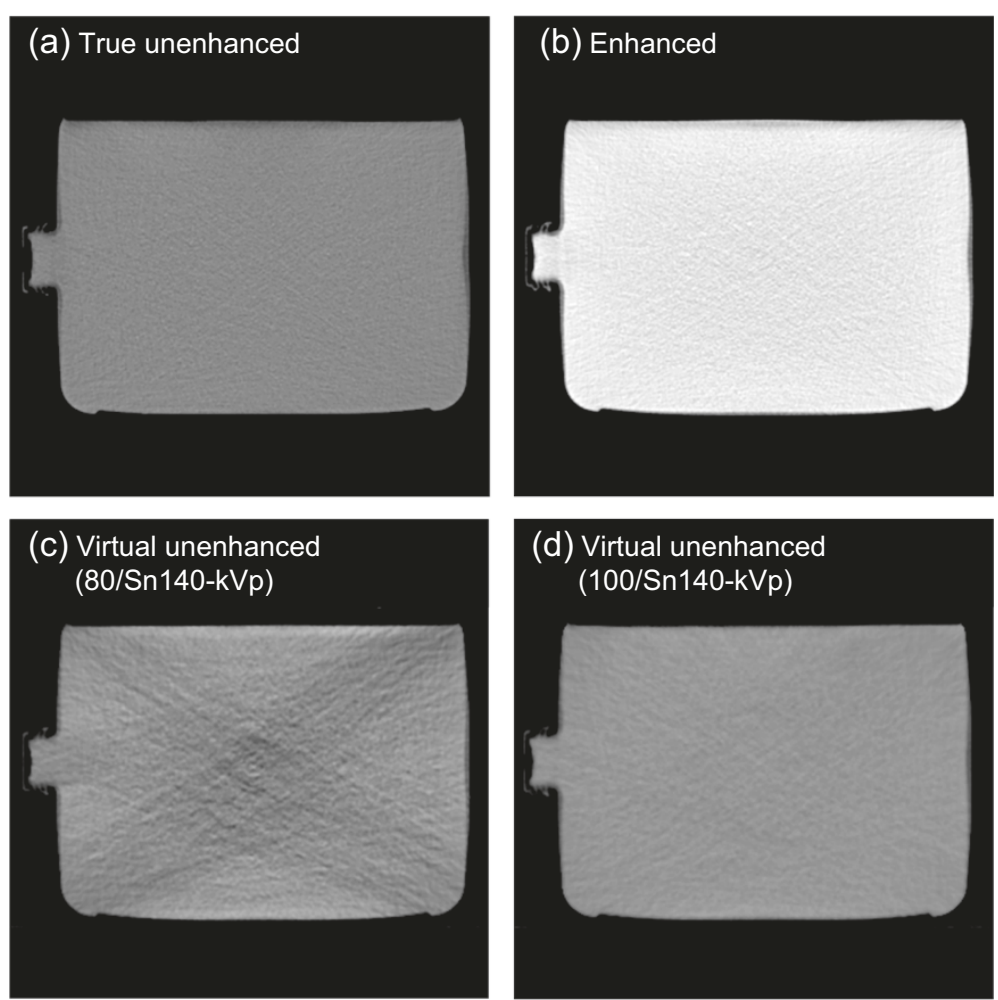

Figure 4 Representative CT images of the phantom for dose calculations. True unenhanced images (a) were obtained using the water phantom. Remaining enhanced images (b), virtual unenhanced (80/Sn140-kVp) images (c), and virtual unenhanced (100/Sn140-kVp) images (d) were obtained using the iodine phantom. The enhanced image (b) shows almost uniform attenuation, indicating no obvious iodine sedimentation. The virtual unenhanced image obtained using the 80/Sn140-kVp protocol shows dark-band artifact and increased image noise, while the image using the 100/Sn140-kVp protocol provided adequate image quality. 


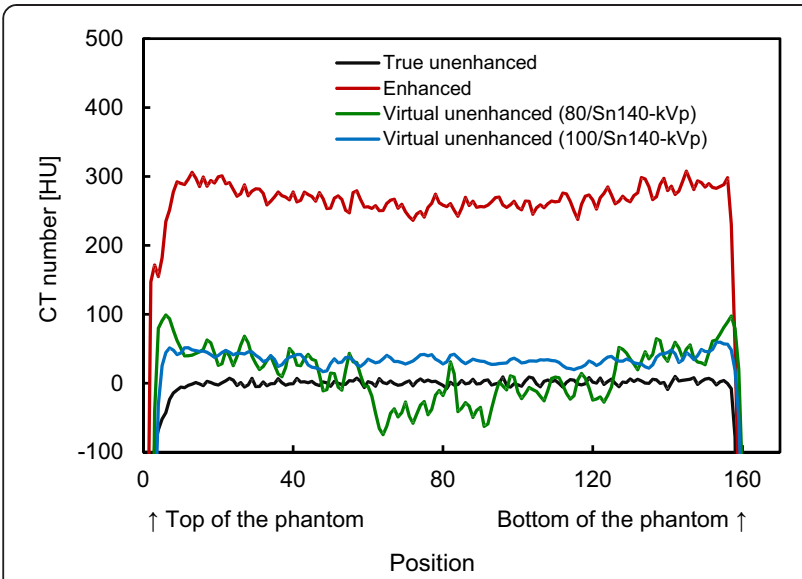

Figure 5 Attenuation profiles. Attenuation values in $\mathrm{HU}$ are plotted against the pixel locations (shown in Figure 2b). The profile from the enhanced image (red line) shows almost uniform distribution of attenuation values with a slight cupping due to beam hardening, indicating no obvious iodine sedimentation. The profile from the virtual unenhanced images using the 100/Sn140-kVp protocol is quite stable, while the profile using the $80 / \mathrm{Sn} 140-\mathrm{kVp}$ protocol was highly fluctuated by dark-band artifacts.

$\mathrm{kVp}$ protocol, were used. Table 3 compares the pass rates of the dose calculations based on the virtual unenhanced and enhanced images. When the virtual unenhanced images were utilized for dose calculations, pass rates greater than $90 \%$ were achieved, while the pass rates were less than approximately $50-60 \%$ when enhanced images were used.

\section{Discussion}

The use of a contrast agent during CT for treatment planning offers the potential benefit of a much higher accuracy in delineating target and critical volumes. However, there is a significant concern that the high atomic number of iodine may introduce errors into dose calculations; the calculated dose may be underestimated, resulting in the delivery of a higher dose than prescribed. This scenario would occur in organs containing an increased iodine concentration. In fact, Shibamoto et al. found that the administration of a contrast agent influenced

Table 2 Attenuation measurements of water and iodine phantoms

\begin{tabular}{lc}
\hline Measurements & Attenuation values $[\mathrm{HU}]$ \\
\hline Water phantom/true unenhanced image & $1.6 \pm 11.0$ \\
lodine phantom/enhanced image & $274.3 \pm 22.5$ \\
lodine phantom/virtual unenhanced & $11.6 \pm 29.2$ \\
$(80 / S n 140-\mathrm{kVp})$ image & \\
lodine phantom/virtual unenhanced & $34.1 \pm 9.9$ \\
$(100 / \mathrm{Sn} 140-\mathrm{kVp})$ image & \\
\hline
\end{tabular}

Note: Attenuation values are mean \pm standard deviation in the region of interest. treatment planning at the upper abdomen, especially when the beams passed through organs with high iodine concentrations such as the liver, spleen, and kidneys [5]. Our present results support their findings. The iodine phantom used in this study presented almost uniformly distributed attenuation values of approximately $270 \mathrm{HU}$ (Figure 4b; Figure 5; Table 2), which approximates that of organs in the upper abdomen on contrast-enhanced CT. As shown in Figures 6, 7 and 8(b), the dose distributions obtained from the enhanced images show increased beam attenuation compared to those from the true unenhanced images (a). The corresponding error maps (Figures 6, 7 and $8 \mathrm{e}$ ) show that the calculated dose obtained from the enhanced images tends to differ from that based on the true unenhanced images as the beam passes through the phantom from the bottom to the top. In Figure 7, for instance, the dose distribution (a) obtained using the true unenhanced images shows a delivery of 72 cGy at the 10$\mathrm{cm}$ depth of the central axis; in contrast, the dose error was at least 4 cGy when the enhanced images were used (e). In this case, the use of the enhanced images leads to erroneous dose delivery greater than $5 \%$ of the prescribed dose. This error is not negligible and would be problematic for treatment planning, especially in the upper abdomen as Shibamoto et al. reported [5].

Our novel approach to solve this issue is to remove the iodine component from contrast-enhanced CT using dual-energy virtual unenhanced imaging. Dual-energy virtual unenhanced imaging has recently become a promising technique in diagnostic $\mathrm{CT}$ to reduce the radiation dose by negating the need for an unenhanced scan [9-11]. Although the clinical potential of dual-energy CT in the field of radiation oncology has not been well investigated to date, several studies have shown that dual-energy CT allows for an accurate estimation of electron density for treatment planning [19-21]. Our study reveals an additional important aspect of its application that could improve the accuracy of radiotherapy treatment planning. Figure 3 shows that virtual unenhanced CT can remove the iodine component from a contrast-enhanced CT image within a wide range of iodine concentrations at $\mathrm{CT}$ number levels greater than the water density, in accordance with previous reports [9-14], including a similar phantom study of iodine solutions [10]. Thus, our study aimed to improve the accuracy of dose calculations from contrast-enhanced CT by removing their iodine components, and the results successfully demonstrated the feasibility of our proposed method. As shown in Figures 6, 7 and 8 , the dose distributions obtained from virtual unenhanced images (c and d) were almost equivalent to those from true unenhanced images (a). The differences in dose calculations between true unenhanced and virtual unenhanced images were extremely close to 0 cGy (f and g), except at the beam edges and dose build-up regions (near 


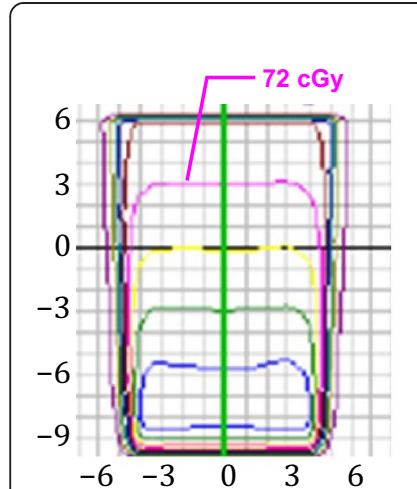

(a) True unenhanced

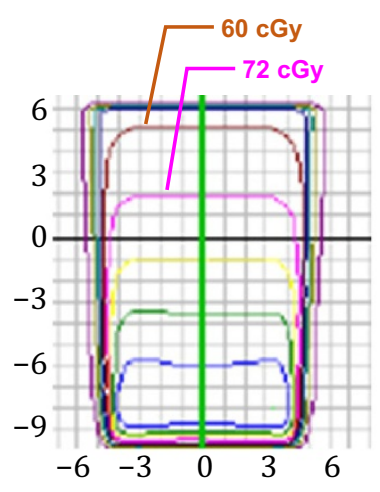

(b) Enhanced

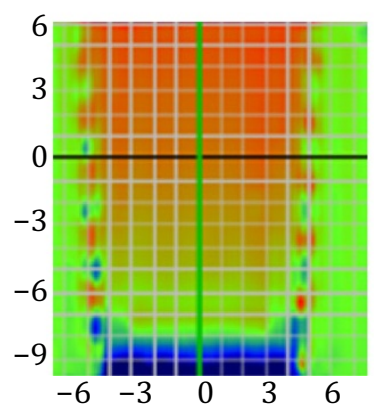

(e) Error in (b)

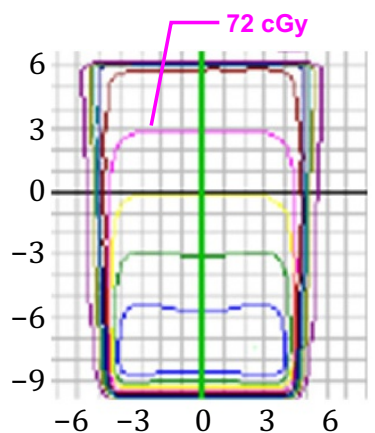

(c) Virtual unenhanced (80 / Sn140 kVp)

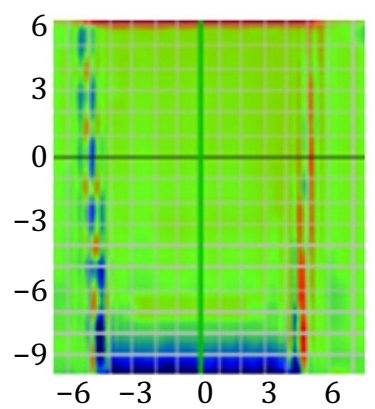

(f) Error in (c)

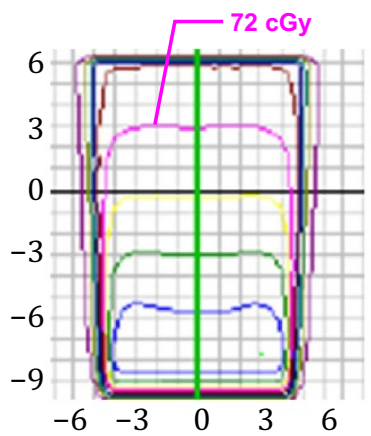

cGp

미요 무을 $\square ?$ 口60 口48 $\square 34$ $\square 12$

\section{(d) Virtual unenhanced (100 / Sn140 kVp)}

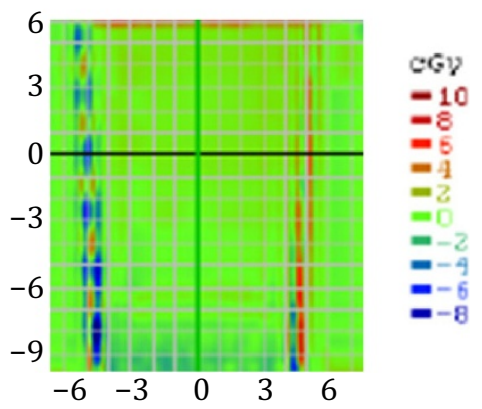

(g) Error in (d)

Figure 6 Planar dose distributions and error maps (energy, $10 \mathrm{MV}$; field size, $10 \times 10 \mathrm{~cm}^{2}$ ). Dose calculations were performed using (a) true unenhanced, (b) enhanced, (c) virtual unenhanced (80/Sn140-kVp), and (d) virtual unenhanced (100/Sn140-kVp) images. The three error (difference) maps correspond to the (e) enhanced, (f) virtual unenhanced (80/Sn140-kVp), and (g) virtual unenhanced (100/Sn140-kVp) images, with the true unenhanced images as the reference. Dose distributions obtained from the virtual unenhanced images are quite similar to dose distributions from the true unenhanced images. In particular, the virtual unenhanced (100/Sn140-kVp) images achieved almost entirely 0-cGy of errors, except at the beam edges.

the bottom of the phantom), which both have high dose gradients. In addition, the use of the $100 / \mathrm{Sn} 140-\mathrm{kVp}$ protocol further improved the accuracy of dose distributions at the dose build-up region. The pass rates of dose calculations (Table 3 ) from the virtual unenhanced images were greater than $90 \%$, whereas those from the enhanced images were less than approximately $50-60 \%$ and thus no longer acceptable.

The virtual unenhanced imaging results in potentially unstable CT numbers when the differences between attenuation values at low- and high-kVp are small. Therefore, the "first-generation" dual-source CT scanner minimized spectral overlap by performing the low-energy scan at $80 \mathrm{kVp}$ [8]. However, the use of an $80-\mathrm{kVp}$ beam brought about an inappropriately low photon rate at the detectors, especially in the abdominal scan, due to a low penetration. Thus, the resultant virtual unenhanced images suffered from increased image noise and artifacts. Although the tin filter could improve spectral separation, the use of an $80-\mathrm{kVp}$ beam would still be problematic. The virtual unenhanced attenuation values of iodine syringes with the 80/Sn140$\mathrm{kVp}$ protocol were indeed more stable than those obtained with the 100/Sn140-kVp protocol at high iodine concentrations (Table 1); this experiment was performed with a small phantom. In fact, the virtual unenhanced $(80 / \mathrm{Sn} 140-\mathrm{kVp})$ images of the iodine phantom, which was used in the second experiment, suffer from increased noise and darkband artifacts (Figure 4,c). The attenuation profile with the $80 / \mathrm{Sn} 140-\mathrm{kVp}$ protocol (Figure 5, green line) fluctuated greatly at the center of the phantom, due to the dark-band artifacts. In contrast, the virtual unenhanced attenuation values using the $100 / \mathrm{Sn} 140-\mathrm{kVp}$ protocol were stable qualitatively (Figure $4 \mathrm{~d}$ ) and quantitatively (Figure 5, blue line). The mean attenuation values of the virtual unenhanced $(100 / \mathrm{Sn} 140-\mathrm{kVp})$ and true unenhanced images were $34.1 \pm 9.9 \mathrm{HU}$ and $1.6 \pm 11.0 \mathrm{HU}$, respectively. This small difference had no effect on dose calculations as shown in Figures 6, 7 and 8. 


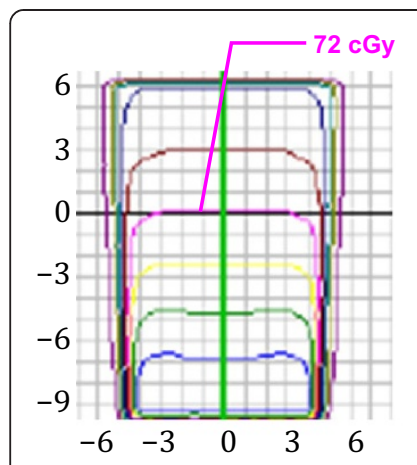

(a) True unenhanced

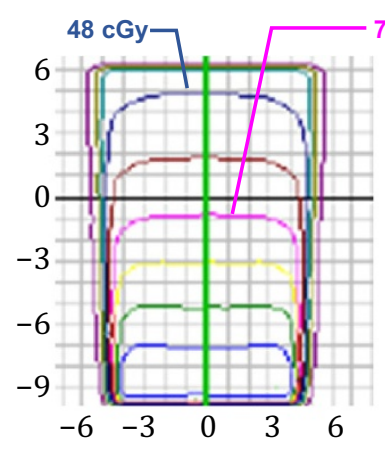

(b) Enhanced

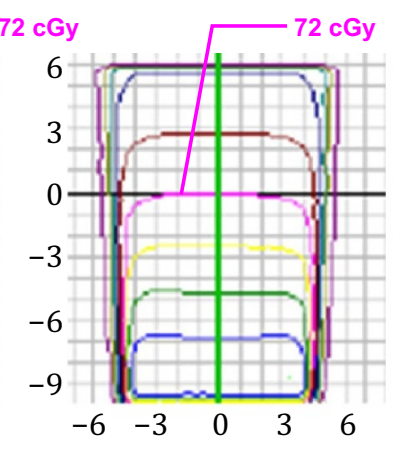

(c) Virtual unenhanced (80 / Sn140 kVp)

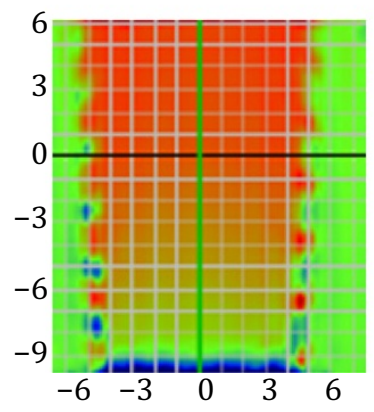

(e) Error in (b)

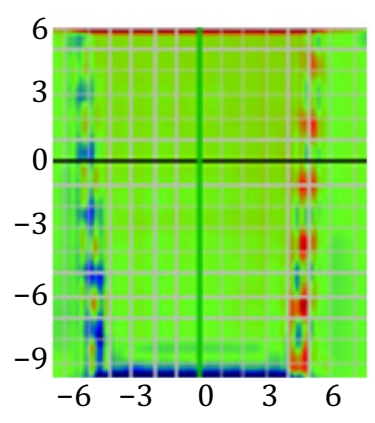

(f) Error in (c)

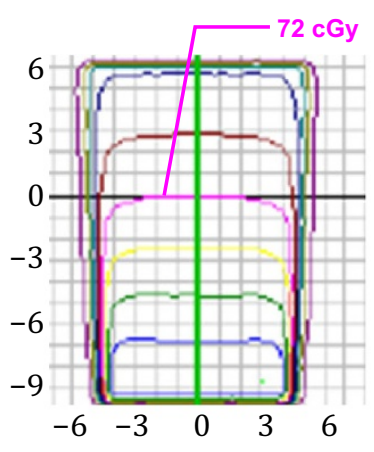

cGy

$\square 120$ 무응 口72 口60 口. 38

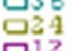

\section{(d) Virtual unenhanced $(100 / \mathrm{Sn} 140 \mathrm{kVp})$}

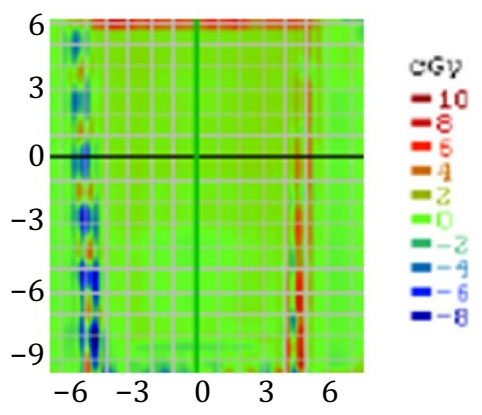

(g) Error in (d)

Figure 7 Planar dose distributions and error maps (energy, $4 \mathbf{~ M V}$; field size, $10 \times 10 \mathbf{c m}^{2}$ ). Dose calculations were performed using (a) true unenhanced, (b) enhanced, (c) virtual unenhanced (80/Sn140-kVp), and (d) virtual unenhanced (100/Sn140-kVp) images. The three error (difference) maps correspond to (e) enhanced, (f) virtual unenhanced (80/Sn140-kVp), and (g) virtual unenhanced (100/Sn140-kVp) images, with the true unenhanced images as the reference. In addition to findings similar to those in Figure 6 , more prominent dose errors are elicited using the enhanced images (e).

The current dual-source virtual unenhanced CT has several known potential limitations, which were investigated by Barrett, et al. [9]. For radiotherapy treatment planning, its relatively smaller field-of-view and susceptibility to motion warrants consideration. The effective field-ofview that can remove iodine components from contrastenhanced images is limited to $322 \mathrm{~mm}$ or less in diameter. This is problematic in larger patients or in more superficial radiotherapy targets because the contrast-enhanced tissue that is located outside the region will remain "enhanced" after the iodine-removal process. This limitation arises from the current scanner configuration, and therefore, it can be alleviated in the future. Also notable is the relatively higher susceptibility to motion in virtual unenhanced imaging, which arises from the $95^{\circ}$ offset of the source-detector systems. The angle offset causes one detector to see a different motion compared to the other detector, and this difference is amplified by the subtraction process. A shorter gantry rotation time can reduce motion artifacts, but the potential for the artifact remains, particularly in the upper-abdominal organs, lung bases, and cardiovascular systems. Another potential limitation of virtual unenhanced imaging is the underestimation of attenuation values of high atomic number materials such as bone. In previous reports [9-11,14], clinical virtual unenhanced images visually demonstrated highly attenuating bone, resembling the normal CT appearance; the iodine removal process would affect the virtual unenhanced attenuation value, depending on the parameters of iodine removal (e.g., "standard tissue" and "fat" CT numbers, "beam-hardening correction" option for iodine, and "relative contrast enhancement ratio" value between low- and high-energy images in "Liver VNC" application). We expect that this underestimation of bone attenuation has little influence on the dose calculation because bone (especially cortical bone) encompasses a relatively small volume within the trunk; however, further investigations evaluating the accuracy and the impact of bone virtual unenhanced attenuation values on dose calculations, optimizing the processing parameters, and (if necessary) developing a correction scheme are needed. 


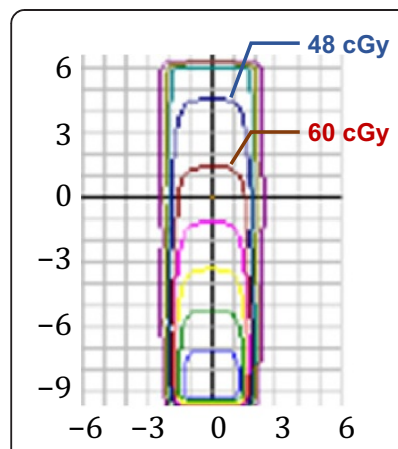

(a) True unenhanced

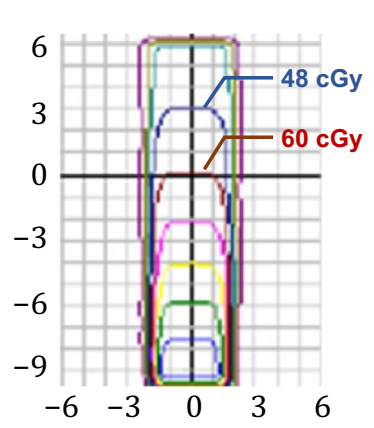

(b) Enhanced

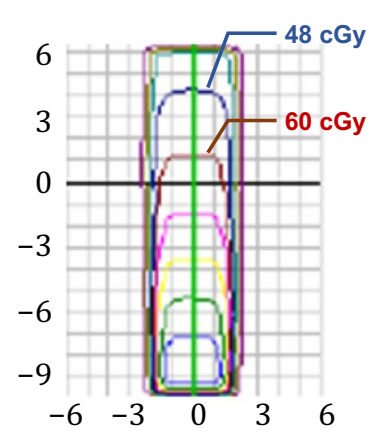

(c) Virtual unenhanced (80 / Sn140 kVp)

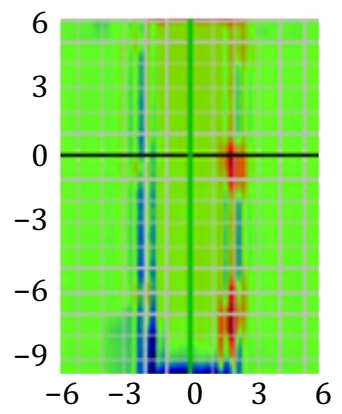

(f) Error in (c)

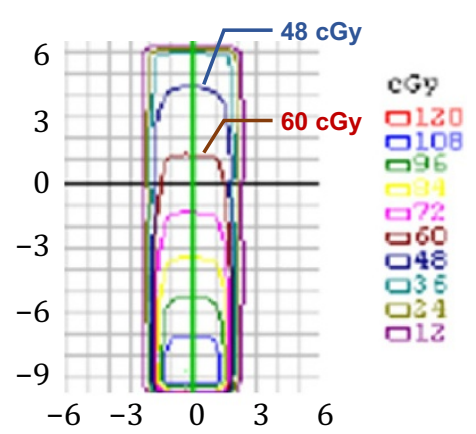

(d) Virtual unenhanced (100 / Sn140 kVp)

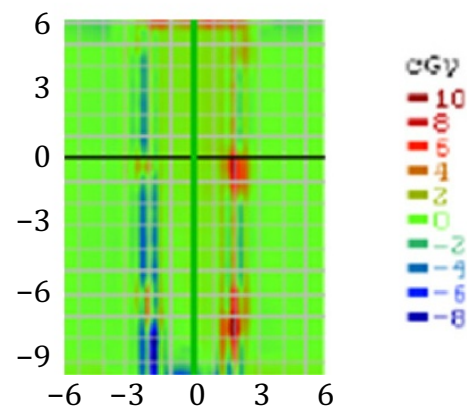

(g) Error in (d)

Figure 8 Planar dose distributions and error maps (energy, $4 \mathrm{MV}$; field size, $\mathbf{4} \times \mathbf{4} \mathrm{cm}^{2}$ ). Dose calculations were performed using (a) true unenhanced, (b) enhanced, (c) virtual unenhanced (80/Sn140-kVp), and (d) virtual unenhanced (100/Sn140-kVp) images. The three error (difference) maps correspond to (e) enhanced, (f) virtual unenhanced (80/Sn140-kVp), and (g) virtual unenhanced (100/Sn140-kVp) images, with the true unenhanced images as the reference. Findings similar to those in Figures 6 and 7 were observed.

This proof-of-concept study is very preliminary, and therefore has several limitations. First, we only conducted simple phantom experiments and did not perform any clinical investigations. Second, the optimal acquisition and reconstruction parameters suitable for applying virtual unenhanced images to radiotherapy treatment planning were not investigated. These limitations are practical in nature; the dual-energy CT scanner used in this study is currently not available for radiotherapy treatment planning. Third, as mentioned above, the reliability of virtual unenhanced attenuation values of high atomic number materials such as bone has not been investigated. Further investigation of these issues is needed to confirm the clinical potential of virtual unenhanced $\mathrm{CT}$ in radiotherapy treatment planning.

\section{Conclusions}

We have proposed a novel strategy to improve the accuracy of radiotherapy treatment planning using dual-energy virtual unenhanced CT. The introduction of contrast agents can improve the delineation of the target and risk organs. The iodine component can be subsequently removed from

Table 3 Pass rates of dose calculations based on the enhanced and virtual unenhanced CT images

\begin{tabular}{|c|c|c|c|c|}
\hline \multirow{3}{*}{ Energy [MV] } & \multirow{3}{*}{ Field size $\left[\mathrm{cm}^{2}\right]$} & \multicolumn{3}{|c|}{ Pass rate [\%] } \\
\hline & & \multirow{2}{*}{ Enhanced } & \multicolumn{2}{|c|}{ Virtual unenhanced } \\
\hline & & & $80 \mathrm{kVp} / \mathrm{Sn} 140 \mathrm{kVp}$ & $100 \mathrm{kVp} / \mathrm{Sn} 140 \mathrm{kVp}$ \\
\hline 10 & $10 \times 10$ & 45.6 & 98.4 & 98.4 \\
\hline 10 & $4 \times 4$ & 59.3 & 98.4 & 99.2 \\
\hline 4 & $10 \times 10$ & 37.1 & 93.7 & 97.9 \\
\hline 4 & $4 \times 4$ & 44.9 & 98.7 & 98.4 \\
\hline
\end{tabular}


contrast-enhanced CT images to provide virtual unenhanced images to perform more accurate dose calculations. Our results have successfully provided the first proof-ofconcept.

\section{Competing interests}

The authors declare that they have no competing interests.

\section{Authors' contributions}

SY conceived of the study, participated in its design and coordination, and drafted the manuscript. SY, TU, TO, HM, RO, MK, TS, and $\mathrm{KO}$ contributed to data acquisition. SY, TU, TO, HM, MK, KM, and KO performed data analysis and interpretation. $\mathrm{TU}, \mathrm{TO}, \mathrm{HM}, \mathrm{RO}, \mathrm{MK}, \mathrm{TS}, \mathrm{KM}$, and $\mathrm{KO}$ helped to finalize the manuscript. All authors read and approved the final manuscript.

\section{Acknowledgements}

This study was supported in part by a Japan Society for the Promotion of Science (JSPS) Grants-in-Aid for Scientific Research (No. 25460751). The authors gratefully acknowledge the assistance of the staff at the Trauma and Acute Critical Care Center (TACCC) of Osaka University Hospital, especially Mr. Takeo Azuma, a senior radiological technologist.

\section{Author details}

'Department of Radiology, Osaka University Hospital, 2-15 Yamadaoka, Suita, Osaka 565-0871, Japan. ${ }^{2}$ Department of Medical Physics and Engineering, Osaka University Graduate School of Medicine, 1-7 Yamadaoka, Suita, Osaka 565-0871, Japan. ${ }^{3}$ Center for Information and Neural Networks, National Institute of Information and Communications Technology, 1-4 Yamadaoka, Suita, Osaka 565-0871, Japan. ${ }^{4}$ Graduate School of Frontier Biosciences, Osaka University, 1-4 Yamadaoka, Suita, Osaka 565-0871, Japan. ${ }^{5}$ Kobe Minimally Invasive Cancer Center, 8-5-1 Minatojima-nakamachi, Chuo-ku, Kobe, Hyogo 650-0046, Japan. ${ }^{6}$ Department of Traumatology and Acute Critical Medicine, Osaka University Graduate School of Medicine, 2-2 Yamadaoka, Suita, Osaka 565-0871, Japan. ${ }^{7}$ Department of Radiation Oncology, Osaka University Graduate School of Medicine, 2-2 Yamadaoka, Suita, Osaka 565-0871, Japan.

Received: 25 March 2014 Accepted: 22 July 2014

Published: 29 July 2014

\section{References}

1. Ramm U, Damrau M, Mose S, Manegold KH, Rahl CG, Böttcher HD: Influence of CT contrast agents on dose calculations in a 3D treatment planning system. Phys Med Biol 2001, 46:2631-2635.

2. Liauw SL, Amdur RJ, Mendenhall WM, Palta J, Kim S: The effect of intravenous contrast on intensity-modulated radiation therapy dose calculations for head and neck cancer. Am J Clin Oncol 2005, 28:456-459.

3. Lees J, Holloway L, Fuller M, Forstner D: Effect of intravenous contrast on treatment planning system dose calculations in the lung. Australas Phys Eng Sci Med 2005, 28:190-195.

4. Weber DC, Rouzaud M, Miralbell R: Bladder opacification does not significantly influence dose distribution in conformal radiotherapy of prostate cancer. Radiother Oncol 2001, 59:95-97.

5. Shibamoto Y, Naruse A, Fukuma H, Ayakawa S, Sugie C, Tomita N: Influence of contrast materials on dose calculation in radiotherapy planning using computed tomography for tumors at various anatomical regions: a prospective study. Radiother Oncol 2007, 84:52-55.

6. Zabel-du Bois A, Ackermann B, Hauswald H, Schramm O, Sroka-Perez G, Huber $P$, Debus J, Milker-Zabel S: Influence of intravenous contrast agent on dose calculation in 3-D treatment planning for radiosurgery of cerebral arteriovenous malformations. Strahlenther Onkol 2009, 185:318-324.

7. Wertz $\mathrm{H}$, Jäkel $\mathrm{O}$ : Influence of iodine contrast agent on the range of ion beams for radiotherapy. Med Phys 2004, 31:767-773.

8. Johnson TR, Krauß B, Sedlmair M, Grasruck M, Bruder H, Morhard D, Fink C, Weckbach S, Lenhard M, Schmidt B, Flohr T, Reiser MF, Becker CR: Material differentiation by dual energy CT: initial experience. Eur Radiol 2007, 17:1510-1517.

9. Barrett T, Bowden DJ, Shaida N, Godfrey EM, Taylor A, Lomas DJ, Shaw AS: Virtual unenhanced second generation dual-source $C T$ of the liver: is it time to discard the conventional unenhanced phase? Eur J Radiol 2012, 81:1438-1445

10. Toepker M, Moritz T, Krauss B, Weber M, Euller G, Mang T, Wolf F, Herold CJ, Ringl H: Virtual non-contrast in second-generation, dual-energy computed tomography: reliability of attenuation values. Eur J Radiol 2012, 81:e398-405.

11. Sommer CM, Schwarzwaelder CB, Stiller W, Schindera ST, Stampfl U, Bellemann N, Holzschuh M, Schmidt J, Weitz J, Grenacher L, Kauczor HU, Radeleff BA: lodine removal in intravenous dual-energy CTcholangiography: is virtual non-enhanced imaging effective to replace true non-enhanced imaging? Eur J Radiol 2012, 81:692-699.

12. Kawai T, Shibamoto Y, Hara M, Arakawa T, Nagai K, Ohashi K: Can dual-energy $\mathrm{CT}$ evaluate contrast enhancement of ground-glass attenuation? Phantom and preliminary clinical studies. Acad Radiol 2011, 18:682-689.

13. Kawai T, Takeuchi M, Hara M, Ohashi K, Suzuki H, Yamada K, Sugimura Y, Shibamoto Y: Accuracy of iodine removal using dual-energy CT with or without a tin filter: an experimental phantom study. Acta Radiol 2013, 54:954-960

14. Kaufmann S, Sauter A, Spira D, Gatidis S, Ketelsen D, Heuschmid M, Claussen $C D$, Thomas C: Tin-filter enhanced dual-energy-CT: image quality and accuracy of CT numbers in virtual noncontrast imaging. Acad Radiol 2013, 20:596-603.

15. De Cecco CN, Darnell A, Macías N, Ayuso JR, Rodríguez S, Rimola J, Pagés M, García-Criado Á, Rengo M, Laghi A, Ayuso C: Virtual unenhanced images of the abdomen with second-generation dual-source dualenergy computed tomography: image quality and liver lesion detection. Invest Radiol 2013, 48:1-9.

16. Primak AN, Ramirez Giraldo JC, Liu X, Yu L, McCollough CH: Improved dual-energy material discrimination for dual-source $\mathrm{CT}$ by means of additional spectral filtration. Med Phys 2009, 36:1359-1369.

17. Primak AN, Giraldo JC, Eusemann CD, Schmidt B, Kantor B, Fletcher JG, McCollough CH: Dual-source dual-energy CT with additional tin filtration: dose and image quality evaluation in phantoms and in vivo. AJR Am J Roentgenol 2010, 195:1 164-1174.

18. Van Dyk J, Barnett RB, Cygler JE, Shragge PC: Commissioning and quality assurance of treatment planning computers. Int J Radiat Oncol Biol Phys 1993, 26:261-273.

19. Ogata T, Ueguchi T, Yagi M, Yamada S, Tanaka C, Ogihara R, Isohashi F, Yoshioka Y, Tomiyama N, Ogawa K, Koizumi M: Feasibility and accuracy of relative electron density determined by virtual monochromatic $\mathrm{CT}$ value subtraction at two different energies using the gemstone spectral imaging. Radiat Oncol 2013, 8:83.

20. Yagi M, Ueguchi T, Koizumi M, Ogata T, Yamada S, Takahashi Y, Sumida I, Akino Y, Konishi K, Isohashi F, Tomiyama N, Yoshioka Y, Ogawa K: Gemstone spectral imaging: determination of $C T$ to ED conversion curves for radiotherapy treatment planning. I Appl Clin Med Phys 2013, 14:173-186.

21. Saito M: Potential of dual-energy subtraction for converting $\mathrm{CT}$ numbers to electron density based on a single linear relationship. Med Phys 2012, 39:2021-2030.

doi:10.1186/1748-717X-9-168

Cite this article as: Yamada et al: Radiotherapy treatment planning with contrast-enhanced computed tomography: feasibility of dual-energy virtual unenhanced imaging for improved dose calculations. Radiation Oncology 2014 9:168. 